\title{
Saturn's Icy Moon Rhea: A Prediction for its Bulk Chemical Composition and Physical Structure at the Time of the Cassini Spacecraft First Flyby
}

\author{
Andrew J. R. Prentice $\mathrm{A}^{\mathrm{A}}$ \\ A School of Mathematical Sciences, Monash University, Melbourne VIC 3800, Australia. \\ Email: andrew.prentice@sci.monash.edu.au \\ Received 2005 November 30, accepted 2006 January 31
}

\begin{abstract}
I report a model for the formation of Saturn's family of mid-sized icy moons to coincide with the first flypast of Rhea by the Cassini spacecraft on 2005 November 26. It is proposed that the moons had condensed from a concentric family of orbiting gas rings that were shed some $4.6 \times 10^{9} \mathrm{yr}$ ago by the proto-Saturnian (hereafter p-Sat) cloud. The p-Sat cloud is made up of gas and residual grains of the gas ring that was shed by the proto-Solar cloud (hereafter PSC) at Saturn's orbit. The bulk of the condensate within this proto-Solar ring accumulates to form Saturn's central core of mass $\sim 10-20 M_{\oplus}\left(M_{\oplus}=\right.$ Earth mass). The process of formation of Saturn's solid core thus provides an opportunity for the $\mathrm{p}$-Sat cloud to become depleted in rock and water ice relative to the usual solar abundances of these materials. Nitrogen, which exists as uncondensing $\mathrm{N}_{2}$ in the PSC and as $\mathrm{NH}_{3}$ in the p-Sat cloud, retains its solar abundance relative to $\mathrm{H}_{2}$. If the depletion factor of solids relative to gas is $\zeta_{\mathrm{dep}}=0.25$, as suggested by the low mass of Rhea relative to solar abundance expectations, the mass-percent ratio of $\mathrm{NH}_{3}$ to $\mathrm{H}_{2} \mathrm{O}$ in the dense p-Sat cloud is 36:64. Numerical and structural models for Rhea are constructed on the basis of a 'cosmogonic' bulk chemical composition of hydrated rock (mass fraction 0.385), $\mathrm{H}_{2} \mathrm{O}$ ice (0.395), and $\mathrm{NH}_{3}$ ice $(0.220)$. It is difficult to construct a chemically differentiated model of Rhea whose mean density matches the observed value $\rho_{\text {Rhea }}=1.23 \pm 0.02 \mathrm{~g} \mathrm{~cm}^{-3}$ for reasonable bounds of the controlling parameters. Chemically homogeneous models can, however, be constrained to match the observed Rhea density provided that the mass fraction of $\mathrm{NH}_{3}$ is permitted to exceed the cosmogonic value by a factor $\zeta_{\mathrm{NH}_{3}}=1.20-1.35$. A large proportion of $\mathrm{NH}_{3}$ in the ice mass inhibits the formation of the dense crystalline phase II of $\mathrm{H}_{2} \mathrm{O}$ ice at high pressure. This may explain the lack of compressional features on the surface of the satellite that are expected as a result of ice II formation in the cooling core. The favoured model of Rhea is chemically uniform and has mass proportions of rock (0.369), $\mathrm{H}_{2} \mathrm{O}$ ice $(0.378)$, and $\mathrm{NH}_{3}$ ice (0.253). The enhancement factor of $\mathrm{NH}_{3}$ lies within the measured uncertainties of the solar abundance of nitrogen. The satellite is very cold and nearly isodense. The predicted axial moment-of-inertia coefficient is $\left[C / M R^{2}\right]_{\text {Rhea }}=0.399 \pm 0.004$.
\end{abstract}

Keywords: Solar system: formation — Sun: Saturn: Rhea - physical data and processes: convection

\section{Introduction}

\subsection{The Cassini Spacecraft Rhea-1 Flypast and Previous Knowledge of Rhea}

On 2005 November 26, the NASA/ESA Cassini Orbiter spacecraft passed within $500 \mathrm{~km}$ of the surface of Saturn's second-largest moon Rhea. The Rhea-1 flypast concludes an exciting series of first-time close encounters with Phoebe, Titan, Enceladus, Hyperion, and Dione. The two remaining close encounters with the mid-sized moons do not occur until late in the Cassini mission. These are with Iapetus in 2007 September and Enceladus in 2008 March. Each of the Cassini close flypasts are extremely important as they provide a wealth of new data on the physical structure and surficial features of the visited moon. Such data provides a valuable opportunity to test ideas for how Saturn and its family of moons may have formed.
It is the primary purpose of this paper to present a model for the bulk chemical composition and internal structure of Rhea, based on the author's modern Laplacian of solar system origin (Prentice 1978a, 1978b, 1984, 2001). Much of what is presently known about Rhea was obtained 25 years ago from the Voyager $1 \& 2$ spacecraft encounters (Smith et al. 1981; Tyler et al. 1981). The surface of Rhea is very heavily cratered and appears ancient. That is, there appears to be little evidence of any recent resurfacing events, such is the case for Enceladus. Parts of the surface do, however, display wispy streaks. These are believed to be associated with faults and fractures caused by early episodes of tectonic activity. So far only $\mathrm{H}_{2} \mathrm{O}$ ice has been detected on the surface (Clark et al. 1984). The absence of $\mathrm{NH}_{3}$ is not surprising owing both to natural sublimation, especially near the equator, and destruction by solar photolysis 
(Lebofsky 1975; Consolmagno \& Lewis 1978). Since the arrival of the Cassini spacecraft at Saturn in 2004 July, estimates of the mean radius and mass of Rhea have been greatly improved. These are $R_{\text {Rhea }}=764 \pm 4 \mathrm{~km}$ and $M_{\text {Rhea }}=$ $(2.306 \pm 0.009) \times 10^{21} \mathrm{~kg}$, respectively. These yield a mean density $\rho_{\text {Rhea }}=1.23 \pm 0.02 \mathrm{~g} \mathrm{~cm}^{-3}$ (Jacobson 2004; Jacobson et al. 2005). It is unknown if the interior of Rhea is a homogeneous mixture of rock and ices or if the satellite has differentiated into a rocky core and icy mantle. The answer to that question will emerge from analysis of the gravity data to be acquired during the Cassini flypast of 2005 November 26.

\subsection{Previous Modelling of Rhea}

Previous attempts to model the physical structure of Rhea have been carried out by Consolmagno \& Lewis (1978), Lupo \& Lewis (1979), Ellsworth \& Schubert (1983), and Consolmagno (1985). All these authors have assumed a bulk chemical composition of rock and water ice in the mass-percent proportions 60:40. These proportions follow from the solar abundance compilation of Ross \& Aller (1976). Of course, major revisions in the estimates of the solar abundances of the elements have occurred since then (Lodders 2003; Asplund et al. 2005). The current best estimate of the percentage proportions of rock to water ice is close to 50:50 (see Table 1). This results in a condensate mean density of $1.5 \mathrm{~g} \mathrm{~cm}^{-3}$ which greatly exceeds $\rho_{\text {Rhea }}$. Even putting this difficulty to one side, no single consensus for the state of Rhea's interior exists (Castillo 2005). Consolmagno's work suggests that the melting of $\mathrm{H}_{2} \mathrm{O}$ and $\mathrm{NH}_{3}$ ices would occur throughout the inner $30 \%$ of the mass of a Rhea-sized moon, so causing differentiation of rock and ice in that part. Since $\mathrm{NH}_{3}$ ice forms a eutectic melt with $\mathrm{H}_{2} \mathrm{O}$ ice at $176 \mathrm{~K}$ (Hogenboom et al. 1997), the migration of this fluid to the surface would cause partial flooding. This might help explain differences in the observed types of cratered terrain.

Ellsworth \& Schubert (1983) argue that solid-state convection of the ice would efficiently transfer radiogenic heat to the surface, thus preventing the melting of

Table 1. Broad chemical composition of proto-Solar material $^{\mathrm{A}}$

\begin{tabular}{llc}
\hline Category & Species & Mass fraction \\
\hline Gases & $\mathrm{H}_{2}$ & 0.710864 \\
& $\mathrm{He}$ & 0.272684 \\
Ices & $\mathrm{Ne} \& \mathrm{Ar}$ & 0.001379 \\
& $\mathrm{H}_{2} \mathrm{O}$ & 0.005368 \\
& $\mathrm{NH}_{3}$ & 0.000966 \\
Rocks & $\mathrm{CH}_{4}$ & 0.003302 \\
Total & $\mathrm{See}$ text & 0.005437 \\
\hline
\end{tabular}

A Derived from the solar elemental abundance data of Lodders (2003)
$\mathrm{H}_{2} \mathrm{O}$ ice. Furthermore, even if $\mathrm{NH}_{3}$ ice were present in the compositional mixture as ammonia monohydrate $\left(\mathrm{NH}_{3} \cdot \mathrm{H}_{2} \mathrm{O}\right.$; Lewis 1972$)$, this is only a minor chemical constituent based on solar abundance considerations. Its melting is thus unlikely to substantially modify the viscosity of the mixture or trigger widespread differentiation. That is, the satellite should remain chemically homogeneous. But one main difficulty with an undifferentiated Rhea-sized moon relates to the formation of the dense phase II of $\mathrm{H}_{2} \mathrm{O}$ ice in the centre, when the satellite cools with the subsidence of radiogenic heating. This event should have left telltale compressional features at the surface. Such features are not observed. The origin and internal structure of Rhea thus remains a mystery.

\section{The Modern Laplacian Theory of Solar System Origin}

It is proposed that the six mid-sized moons of Saturn Mimas, Enceladus, Tethys, Dione, Rhea, and Iapetus condensed from a concentric family of orbiting gas rings that were shed by the $\mathrm{p}$-Sat cloud. Gas ring shedding is a central feature of the modern Laplacian theory of solar system origin (Prentice 1978a, 1978b, 1984, 2001). It is the means by which the PSC and proto-planetary clouds of Jupiter, Saturn, Uranus, and Neptune dispose of excess spin angular momentum during gravitational contraction. The mean orbital radii $R_{n}$ and masses $m_{n}$ of the sequence of gas rings ( $n=0,1$, $2,3 \ldots$ ) satisfy Eqn (1).

$$
\frac{R_{n-1}}{R_{n}} \approx\left[1+\frac{m_{n}}{M_{n} f_{n}}\right]^{2}
$$

Here $M_{n}$ and $f_{n}$ denote the residual mass and moment of inertia factor of the cloud after shedding the $n$-th gas ring. If the cloud contracts uniformly, so that both $f_{n}$ and $m_{n} / M_{n}$ stay constant, then $R_{n-1} / R_{n}$ is constant also. That is, the orbital radii $R_{n}(n=0,1,2,3 \ldots)$ form a geometric sequence.

We apply Eqn (1) to the p-Sat cloud and choose $f=$ $0.01, M_{n} \approx M_{\text {Sat }}=5.685 \times 10^{26} \mathrm{~kg}$, and $\left\langle R_{n-1} / R_{n}\right\rangle=$ 1.30 , which is the observed mean orbital distance ratio from Mimas through to Rhea. This yields $m_{n}=8.0 \times$ $10^{23} \mathrm{~kg}$. We can use this value to estimate the expected mass of Rhea based on the total mass of condensate (rock, $\mathrm{H}_{2} \mathrm{O}$ ice, and $\mathrm{NH}_{3}$ ice) in the p-Sat gas ring. Table 1 gives the mass fractions of the broad chemical constituents that can be formed from a gas of solar composition. It is computed on the basis of the proto-solar elemental abundance compilation of Lodders (2003). In constructing this table it is assumed that all rock-like elements have been sequestered into oxides $\left(\mathrm{MgO}, \mathrm{SiO}_{2}, \mathrm{Al}_{2} \mathrm{O}_{3}, \mathrm{Fe}_{3} \mathrm{O}_{4}, \mathrm{CaO}, \mathrm{TiO}_{2}, \mathrm{Na}_{2} \mathrm{O}, \mathrm{K}_{2} \mathrm{O}\right.$, $\mathrm{Cr}_{2} \mathrm{O}_{3}, \ldots$ ), sulfides (FeS, NiS, MnS, ZnS), and halides $\left(\mathrm{NaCl}, \mathrm{CaF}_{2}\right)$. Sulfide and halide formation precede that of the oxides. All residual oxygen is used to form $\mathrm{H}_{2} \mathrm{O}$. Also all nitrogen in the p-Sat cloud is present as $\mathrm{NH}_{3}$ 
(Prinn \& Fegley 1981). Assuming then that Rhea consists of rock, $\mathrm{H}_{2} \mathrm{O}$ ice, and $\mathrm{NH}_{3}$ ice in solar proportions, the total mass of condensate is $9.4 \times$ $10^{21} \mathrm{~kg}$. This mass is approximately $4 M_{\text {Rhea }}$. We propose that the shortfall in the observed Rhea mass relative to solar elemental abundance expectation is a natural consequence of the formation of Saturn's rock/ ice core of mass $M_{\text {core }} \approx 10-20 M_{\oplus}$. The accretion of this core exhausted most of the condensed chemical species within the proto-Solar gas ring at Saturn's orbit. It is the residual material of this ring (mostly $\mathrm{H}_{2}$ and $\mathrm{He}$ ) that is later captured by the dense planetary core to form the p-Sat cloud.

Let $\zeta_{\text {dep }}$ denote the depletion factor, relative to solar expectation, in the abundance of those elements in the $\mathrm{p}$-Sat cloud that first condense as solids in the PSC gas ring. This includes all rock-like elements and that component of total oxygen that does not stay in the gas as $\mathrm{H}_{2} \mathrm{O}$ vapour, $\mathrm{CO}$, or $\mathrm{CO}_{2}$ (see below and Prentice 1996a, 2001). The mass fraction of element $i$ in the p-Sat cloud is then

$$
X_{i, \text { Sat }}=\zeta_{\mathrm{dep}} X_{i, \text { Sun }}
$$

Guided by the shortfall of Rhea's mass, we propose that $\zeta_{\text {dep }}=0.25$. Table 2 gives the mass fractions of the broad chemical constituents of the p-Sat cloud that now follow for this choice of $\zeta_{\text {dep }}$. In constructing Table 2, we observe that any element that is not condensed in the proto-Solar gas ring retains its solar abundance proportion. This includes the noble gases and nitrogen, which exists solely as $\mathrm{N}_{2}$ in the PSC. Also carbon is distributed between $\mathrm{CH}_{4}$ (number fraction 0.830$), \mathrm{C}_{(\mathrm{s})}(0.143), \mathrm{CO}_{2}$ (0.013), and $\mathrm{CO}(0.014)$. Here $\mathrm{C}_{(\mathrm{s})}$ means graphite. These numbers emerge from the numerical computation of the gravitational contraction of the PSC that is considered in Section 3

\section{Models for the Proto-Solar and Proto-Saturnian Clouds}

\subsection{Supersonic Turbulent Stress and the Structure of the Proto-Solar Cloud}

In order to determine the bulk chemical composition of the native icy moons of Saturn, it is first necessary to construct a numerical model for the PSC.

Table 2. Broad chemical composition of the protoSaturnian cloud for $\zeta_{\text {dep }}=0.25$

\begin{tabular}{llc}
\hline Category & Species & Mass fraction \\
\hline Gases & $\mathrm{H}_{2}$ & 0.716891 \\
& $\mathrm{He}$ & 0.274958 \\
& $\mathrm{Ne} \& \mathrm{Ar}$ & 0.001391 \\
Ices & $\mathrm{H}_{2} \mathrm{O}$ & 0.001460 \\
& $\mathrm{NH}_{3}$ & 0.000974 \\
& $\mathrm{CH}_{4}$ & 0.002955 \\
Rocks & $\mathrm{Same}$ & 0.001371 \\
Total & & 1.000000 \\
\hline
\end{tabular}

This calculation will yield the thermochemical and compositional state of the gas that makes up the p-Sat cloud. Now in order for the contracting PSC to dispose of its excess spin angular momentum in gas rings, it is necessary that the interior of the cloud be pervaded by a large radial turbulent stress $p_{\text {turb }}$ arising from strongly supersonic, thermal convective motions (Prentice 1973; Prentice \& Dyt 2003). For a non-rotating structure, this stress is given by $p_{\text {turb }}=\beta(r) \rho(r) G M(r) / r$. Here $\rho=\rho(r)$ is the local gas density, $M(r)$ is the mass interior to radius $r$, and $\beta=\beta(r)$ is the turbulence parameter. $G$ is the gravitation constant. The total pressure at each point is $p_{\text {turb }}+p_{\text {gas }}$, where $p_{\text {gas }}=\rho R_{\mathrm{g}} T / \mu$ is the gas pressure, $T$ is the temperature, $\mu$ is the mean molecular weight, and $R_{\mathrm{g}}$ is the gas constant.

Each non-rotating model of given surface radius $R_{\mathrm{S}}$ and total mass $M_{\mathrm{s}}=M\left(R_{\mathrm{s}}\right)$ has an adiabatic core of radius $r_{0}$ inside which $\beta=\beta_{0}$, a constant. The surface of the cloud is defined where the dimensionless temperature function $\theta \cong \mu_{\mathrm{c}} T(r) / \mu T_{\mathrm{c}}=\theta_{\mathrm{s}}$, a constant, and $\mathrm{c}$ refers to the centre. The core itself consists of an inner zone of radius $r_{1}$ in which all hydrogen is taken to be $\mathrm{H}_{1}$ (or $\mathrm{H}^{+}$) and an outer zone in which it is all $\mathrm{H}_{2}$. Lastly, the core is surrounded by a superadiabatic envelope of polytropic index $n_{\mathrm{t}}=-1$ in which $\beta$ falls to 0 as $\theta \rightarrow \theta_{\mathrm{s}}$ according to Eqn (3).

$$
\beta=\beta_{0}\left[\frac{\theta-\theta_{\mathrm{s}}}{\theta_{0}-\theta_{\mathrm{s}}}\right] \quad \theta_{0}=\frac{\mu_{\mathrm{c}} T\left(r_{0}\right)}{\mu_{0} T_{\mathrm{c}}}
$$

Rotation is included using the atmospheric approximation (Prentice 1978a, 1978b). If the controlling parameters $\beta_{0}, \theta_{0}, \theta_{\mathrm{s}}$, and $n_{\mathrm{t}}$ stay constant during the contraction, the PSC sheds gas rings whose mean orbital radii $R_{n}(n=0,1,2,3 \ldots)$ form a nearly geometric sequence. The initial cloud mass $M_{\mathrm{i}}$ is chosen so that the final cloud mass is $M_{\text {Sun }}=1.98892 \times 10^{30} \mathrm{~kg}$. Setting $\theta_{\mathrm{s}}=0.002272, F_{\mathrm{s}}=\theta_{0} / \theta_{\mathrm{s}}=8.534$, and $\beta_{0}=0.1206$ ensures (a) that the mean orbital spacings of the gas rings from Jupiter to Mercury matches the observed planetary spacings, (b) that the condensate bulk density $\rho_{\text {cond }}$ at Mercury's orbit results in a planet of Mercury's physical radius whose mean density equals the observed value, namely $5.43 \pm 0.01 \mathrm{~g} \mathrm{~cm}^{-3}$ (Anderson et al. 1987), and (c) that only a fraction $\phi_{\mathrm{H}_{2} \mathrm{O}}=0.700$ of the total $\mathrm{H}_{2} \mathrm{O}$ at Jupiter's orbit condenses out. This ensures a composition for the proto-Jovian cloud that can account for the observed 55:45 rock-to-ice mass-percent ratios in Ganymede and Callisto (Prentice 2001).

At Saturn's orbit, where the PSC gas ring temperature is $T_{n}=94 \mathrm{~K}$ and the mean orbit pressure is $p_{n}=4.9 \times 10^{-8} \mathrm{MPa}$, the condensate consists of rock (mass fraction 0.4923$)$, water ice $(0.4739)$, and graphite (0.0338). The rock is almost anhydrous and has mean density $3.668 \mathrm{~g} \mathrm{~cm}^{-3}$ at $76 \mathrm{~K}$ and $0.1 \mathrm{MPa}$. The total fraction of the water vapour that is condensed is $\phi_{\mathrm{H}_{2} \mathrm{O}}=$ 0.974 . The condensate mean density at the present-day blackbody temperature at Saturn, namely $T_{\text {Sat }}=76 \mathrm{~K}$, is 
$\rho_{\text {cond }}=1.5226 \mathrm{~g} \mathrm{~cm}^{-3}$. This value for $T_{\text {Sat }}$ assumes a mean surface albedo of 0.5 .

\subsection{Gravitational Contraction of the Proto-Saturnian Cloud}

In 2005 February, the Cassini Orbiter discovered that Enceladus has a mean density $\rho_{\text {Enc }}=1.60 \pm 0.02 \mathrm{~g} \mathrm{~cm}^{-3}$ (Rappaport et al. 2005). This is about $60 \%$ higher than a value of $1.00 \mathrm{~g} \mathrm{~cm}^{-3}$ that had been predicted on the basis of a model for the formation of the Saturn system that had been put forward prior to the Cassini flypast (Prentice 2005a). The primary aim of this pre-Cassini model was to explain the low mean density of Tethys, namely $\rho_{\text {Tethys }}=0.99 \pm 0.01 \mathrm{~g} \mathrm{~cm}^{-3}$, relative to its more distant neighbour Dione.

According to Prentice (2005a), when the equatorial radius $R_{\mathrm{e}}$ of the p-Sat cloud shrinks inside the orbit of Dione, say a radius $R_{\mathrm{e}}=5 R_{\mathrm{Sat}}$, the central planetary core of mass $M_{\text {core }} \approx 15 M_{\oplus}$ releases a substantial quantity of its only volatile constituent, namely $\mathrm{H}_{2} \mathrm{O}$, into the turbulent $\mathrm{p}$-Sat envelope. Initially the water content of the envelope relative to the solar abundance expectation is $W_{\mathrm{H}_{2} \mathrm{O}}=\zeta_{\text {dep }}=0.25$. If some $15 \%$ to $20 \%$ of the $\mathrm{H}_{2} \mathrm{O}$ content of the planetary core is released, the value of $W_{\mathrm{H}_{2} \mathrm{O}}$ rises from 0.25 to 3.0 . This means that the condensate at the orbit of Tethys now consists mostly of water ice (see Tables 3 and 4). It has a mean density that matches the observed value of Tethys.

\subsection{A Refinement to the p-Sat Cloud Model Following} the Cassini Close Flypast of Enceladus

To explain the higher than expected density of Enceladus found by Cassini, it is proposed that the two innermost moons Mimas and Enceladus (and to a lesser extent Tethys) may have initially condensed closer to Saturn than where they are today. These moons then experienced substantial outward radial migration, most possibly as a result of strong tidal action exerted by the young rapidly rotating planet. If Enceladus were to have condensed at orbital distance of approximately $3.25 R_{\text {Sat }}$, rather than at its present distance of $3.951 R_{\text {Sat }}$, where $R_{\text {Sat }}=60268 \mathrm{~km}$, the temperature of its formative gas ring (i.e. the quantity $T_{n}{ }^{*}$ shown in Table 3 and Figure 1) rises from $260 \mathrm{~K}$ to $300 \mathrm{~K}$. This value is just below the local condensation temperature of $\mathrm{H}_{2} \mathrm{O}$ on the mean orbit of the gas ring, namely $T_{\mathrm{H}_{2} \mathrm{O}}=307 \mathrm{~K}$. This means that most of the water content of the gas ring remains in the vapour phase. As a result, the proportion of rock to water in the condensate is greatly enhanced. Also, because the gas pressure $p_{n}=2.45 \mathrm{MPa}$ on the mean orbit is so large, all of the condensing $\mathrm{H}_{2} \mathrm{O}$ is in the liquid state. That is, when Enceladus formed it was a globe of pure water surrounding a rocky core (Prentice 2005b).

Lastly, if we assume that the orbital radii $R_{\text {Rhea }}$ and $R_{\text {Dione }}$ of Rhea and Dione were uninfluenced by tidal action, then the present ratio $R_{\text {Rhea }} / R_{\text {Dione }}=1.3967$ can be used to estimate the initial orbital radii $R_{n, \mathrm{i}}$ of each satellite prior to tidal migration. We assume that these radii define

Table 3. Properties of the proposed family of gas rings shed by the proto-Saturnian cloud

\begin{tabular}{lccccccccc}
\hline Moon & $n$ & $R_{n}\left[R_{\text {Sat }}\right]$ & $R_{n, \mathrm{i}}\left[R_{\text {Sat }}\right]$ & $M_{n}\left[M_{\mathrm{Sat}}\right]$ & $p_{n}[\mathrm{MPa}]$ & $T_{n}{ }^{*}[\mathrm{~K}]$ & $T_{\mathrm{H}_{2} \mathrm{O}}[\mathrm{K}]$ & $T_{\mathrm{NH}_{3}}[\mathrm{~K}]$ & $T_{\mathrm{CH}_{4} \cdot 6 \mathrm{H}_{2} \mathrm{O}}[\mathrm{K}]$ \\
\hline Mimas & 7 & 3.080 & 2.777 & 1.0040 & 3.36 & 317 & 328 & 172 & 145 \\
Enceladus & 6 & 3.951 & 3.250 & 1.0073 & 2.45 & 300 & 307 & 170 & 142 \\
Tethys & 5 & 4.890 & 4.484 & 1.0152 & 0.787 & 222 & 288 & 161 & 132 \\
Dione & 4 & 6.262 & 6.262 & 1.0231 & 0.265 & 170 & 245 & 154 & 123 \\
Rhea & 3 & 8.746 & 8.746 & 1.0311 & 0.0809 & 129 & 234 & 147 & 115 \\
Iapetus & 2 & 12.20 & 12.20 & 1.0391 & 0.0241 & 101 & 224 & 140 & 108 \\
? & 1 & 17.00 & 17.00 & 1.0472 & 0.00696 & 83 & 215 & 134 & 102 \\
Hyperion & 0 & 24.29 & 24.29 & 1.0559 & 0.00176 & 74 & 205 & 128 & 95 \\
\hline
\end{tabular}

Table 4. Condensate bulk composition and mean density

\begin{tabular}{lccccccccc}
\hline Moon & $R_{n, \mathrm{i}}\left[R_{\mathrm{Sat}}\right]$ & $W_{\mathrm{H}_{2} \mathrm{O}}$ & $X_{\text {rock }}$ & $X_{\mathrm{H}_{2} \mathrm{O}}$ & $X_{\mathrm{NH}_{3}}$ & $X_{\mathrm{CH}_{4}}$ & $\rho_{\text {rock }}\left[\mathrm{g} \mathrm{cm}^{-3}\right]$ & $\rho_{\text {cond }}\left[\mathrm{g} \mathrm{cm}^{-3}\right]$ & $\rho_{\text {obs }}\left[\mathrm{g} \mathrm{cm}^{-3}\right]$ \\
\hline Mimas & 2.777 & 6.5 & 0.277 & $0.723^{\mathrm{A}}$ & 0 & 0 & 3.146 & 1.160 & $1.17 \pm 0.04$ \\
Enceladus & 3.250 & 3.0 & 0.574 & $0.426^{\mathrm{A}}$ & 0 & 0 & 3.146 & 1.566 & $1.60 \pm 0.02$ \\
Tethys & 4.484 & 3.0 & 0.088 & 0.912 & 0 & 0 & 3.146 & 0.995 & $0.96 \pm 0.01$ \\
Dione & 6.262 & 0.25 & 0.494 & 0.506 & $5 \times 10^{-5} \mathrm{~B}$ & 0 & 3.154 & 1.431 & $1.47 \pm 0.02$ \\
Rhea & 8.746 & 0.25 & 0.385 & 0.395 & 0.220 & 0 & 3.154 & 1.249 & $1.23 \pm 0.01$ \\
Iapetus & 12.20 & 0.25 & 0.351 & 0.360 & 0.233 & 0.056 & 3.154 & 1.139 & $1.11 \pm 0.04$ \\
? & 17.00 & 0.25 & 0.338 & 0.367 & 0.238 & 0.057 & 3.491 & 1.138 & - \\
Hyperion & 24.29 & 0.25 & 0.333 & 0.370 & 0.240 & 0.057 & 3.637 & 1.137 & $0.58 \pm 0.11$ \\
\hline
\end{tabular}

${ }^{\text {A Liquid water. }}{ }^{\text {B }}$ Mass fraction of $\mathrm{NH}_{3}$ tied up as $\mathrm{NH}_{3} \cdot \mathrm{H}_{2} \mathrm{O}$. 


\section{CHEMICAL CONDENSATION SEQUENCE}

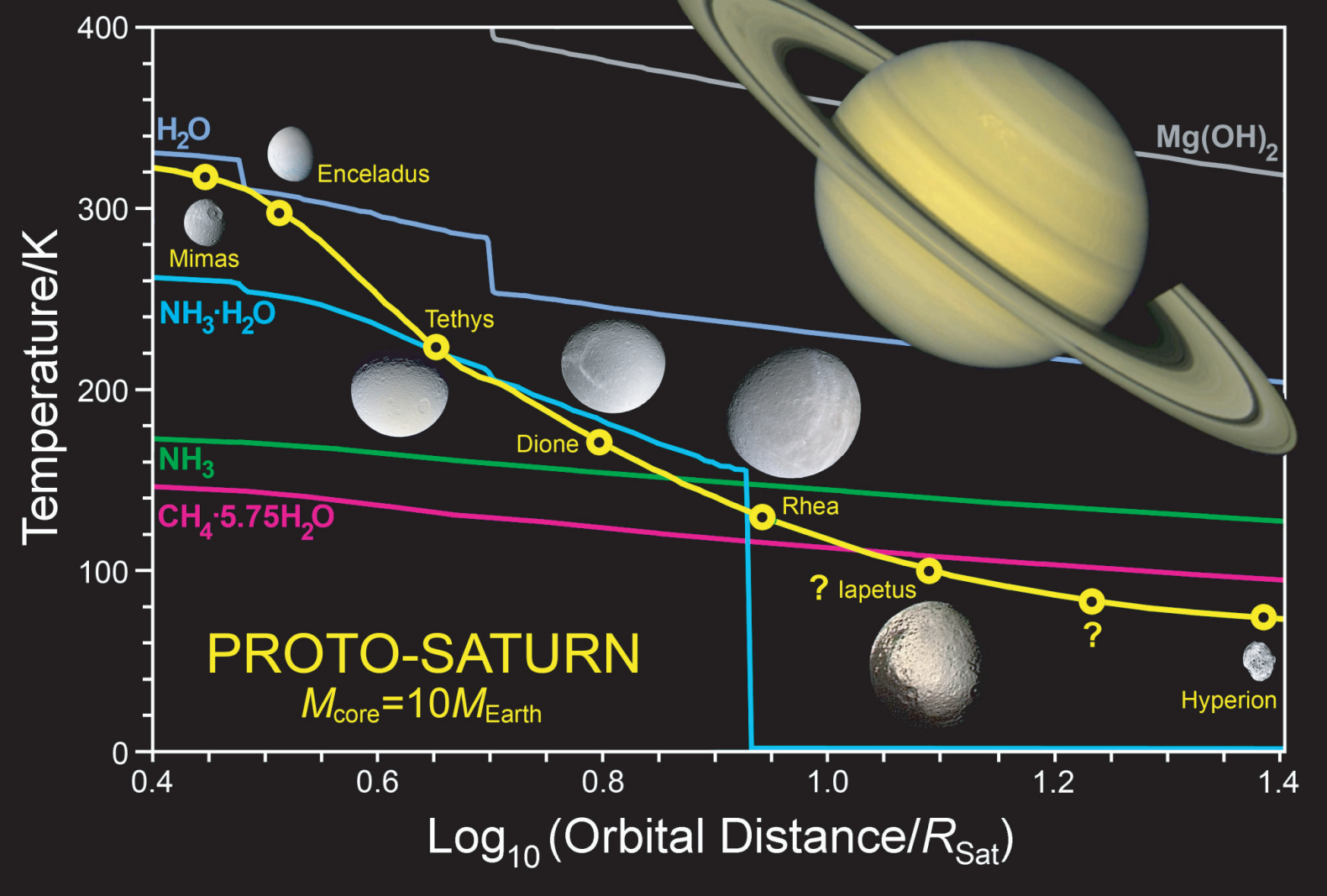

Figure 1 The heavy yellow curve in this diagram gives the temperature of a gas ring at the moment of detachment from the contracting proto-Saturnian cloud. It is calculated against the present orbital radius that the ring would have if it existed today, allowing for secular expansion due to mass loss from the p-Sat cloud. The unit of radius is $R_{\mathrm{Sat}}=60268 \mathrm{~km}$. The temperatures $T_{n}$ of the gas rings from which Saturn's native moons condensed are also plotted (open circles) against their present orbital distances $R_{n}(n=0,1,2,3 \ldots$ ). The other lines in the Figure give the condensation temperatures $T_{i}$ of the principal ice species $\{i\}$ as well as that of the copious rocky constituent brucite. These temperatures are calculated for the gas pressure $p_{n}$ on the mean orbit of the gas ring. The values of $T_{i}, R_{n}$, and $p_{n}$ for each of the satellites are shown in Table 3. The abrupt changes in $T_{\mathrm{H}_{2} \mathrm{O}}$ at orbital distances $3 R_{\text {Sat }}$ and $5 R_{\text {Sat }}$ are due to an enhancement in the water vapour content of the p-Sat envelope. This occurs through a proposed release of $\mathrm{H}_{2} \mathrm{O}$ by Saturn's core of mass $M_{\text {core }}=10 M_{\oplus}$, as discussed in the text. The ratio $W_{\mathrm{H}_{2} \mathrm{O}}$ of the water abundance relative to solar expectation is given in Table 3 .

the mean orbital radii of the family of gas rings shed by the p-Sat cloud. Allowance also has to be made for orbital expansion due to the steady loss of mass of the p-Sat cloud during its gravitational contraction from initial equatorial radius $30 R_{\text {Sat }}$ to present size. That is, as $M_{n}$ is the mass of the cloud at the time of detachment of the $n$-th ring, the initial mean orbital radius of the $n$-th ring is actually $R_{n, \mathrm{i}}{ }^{\prime}=$ $R_{n, \mathrm{i}} M_{\mathrm{Sat}} / M_{n}$. This factor is taken into account in the calculations but for the sake of simplicity only the values of $R_{n, \mathrm{i}}$ are shown in Table 3. I also note that initial orbital distance $R_{n, \mathrm{i}}$ of Enceladus is strictly $3.21 R_{\text {Sat }}$. This difference, however, is not important at this stage. Likewise for Hyperion $(n=0)$, a strict adherence to the geometric spacing law yields $R_{0, \mathrm{i}}=23.8 R_{\text {Sat }}$. This barely differs from the observed orbital distance $R_{0}=24.29 R_{\text {Sat }}$, which is adopted in Table 3. Iapetus is assumed to have formed at the $R_{0, \mathrm{i}}=12.2 R_{\text {Sat }}$ position, prior to being displaced to its present orbit as a consequence of Titan's dynamical capture from a solar orbit (Prentice 1984, 2004a, 2004b). Likewise, the moon that once existed at about $17.0 R_{\text {Sat }}$ is assumed to have been destroyed collisionally by Titan. Much of the material of that former Iapetus-sized moon lies buried in Titan's upper mantle and may be the source of Titan's $\mathrm{N}_{2} /$ $\mathrm{CH}_{4}$ atmosphere.

\subsection{Specification of the New p-Sat Cloud Model Parameters}

Having described what is expected to be achieved by the new p-Sat cloud model, I now specify the values of the parameters $\beta_{0}, \theta_{0}$, and $F_{\mathrm{s}}\left(=\theta_{0} / \theta_{\mathrm{s}}\right)$. First, the value $\theta_{\mathrm{s}, \mathrm{Sat}}=0.004822$ ensures that the ratio of the orbital radii of the gas rings shed at the orbital distances of Rhea and Dione matches the observed ratio, namely 1.3967. Next, the parameter $\beta_{0, \text { Sat }}$ is standardized against the PSC value $\beta_{0, \text { Sun }}$, assuming a linear dependence on the difference $F_{\mathrm{s}}-1$. That is, we assume

$$
\beta_{0, \text { Sat }}=\beta_{0, \text { Sun }}\left[\frac{F_{\mathrm{s}, \text { Sat }}-1}{F_{\mathrm{s}, \text { Sun }}-1}\right]
$$


This assumption has a good physical basis since the strength of turbulent stress depends on the temperature contrast between the value at the base of the superadiabatic convecting layer and that at the surface (Prentice \& Dyt 2003). If $F_{\mathrm{s} \text {, Sat }}-1=0$, all convection ceases. This leaves $F_{\mathrm{s}}=F_{\mathrm{s}, \mathrm{Sat}}$ as the residual free parameter of the model. Now $F_{\text {s }}$ controls the absolute scaling of the gas ring temperature distribution $T_{n}$. If background heating due to the early Sun is ignored and the p-Sat cloud contracts homologously, we have $T_{n} \approx$ $A\left(F_{\mathrm{s}}\right) / R_{n}$ where the constant $A\left(F_{\mathrm{s}}\right)$ depends on $F_{\mathrm{s}}$. Taking into account the radiant energy at Saturn's orbit due to the zero-age main sequence Sun, whose luminosity is $0.7 L_{\text {Sun }}$ (Bahcall, Pinsonneault, \& Basu 2001), the true gas ring temperature is

$$
T_{n}^{*} \approx\left(T_{n}^{4}+0.7 T_{\text {Sat }}^{4}\right)^{0.25}
$$

The mean density $\rho_{\text {cond }}$ of the condensate at the orbit of Enceladus is extremely sensitive to the choice of $F_{\mathrm{s}}$ since the temperature $T_{n}{ }^{*}$ is so close to the $\mathrm{H}_{2} \mathrm{O}$ condensation line. Choosing $F_{\mathrm{s}}=5.6$ we find $\rho_{\text {cond,Enc }}=1.43 \mathrm{~g} \mathrm{~cm}^{-3}$, whereas for $F_{\mathrm{s}}=5.4$ we get $\rho_{\text {cond,Enc }}=1.77 \mathrm{~g} \mathrm{~cm}^{-3}$, allowing for freezing of the $\mathrm{H}_{2} \mathrm{O}$. For $F_{\mathrm{s}}=5.5$, the density of a frozen Enceladus nearly matches the observed value of $1.60 \mathrm{~g} \mathrm{~cm}^{-3}$. The mean density of the condensate at the orbit of Dione is unchanged as $F_{\mathrm{s}}$ varies from 5.4 to 5.6. The mean density at Rhea's orbit also barely changes. It decreases from 1.250 to 1.247 as $F_{\mathrm{s}}$ increases from 5.4 to 5.6. We therefore choose $F_{\mathrm{s}, \text { Sat }}=5.5$.

\subsection{Specification of the State of Saturn's Central Core and the Other Parameters of the p-Sat Cloud Model}

The remaining aspect of the $\mathrm{p}$-Sat cloud model to specify is the physical state of the central rock/ice core of Saturn. The mass of this core is taken to be $M_{\text {core }}=$ $10 M_{\oplus}$. For cloud equatorial radii $R_{\mathrm{e}} \leq 3 R_{\text {Sat }}$, the density of the core is chosen to be $\rho_{\text {core }}=3.25 \mathrm{~g} \mathrm{~cm}^{-3}$. It is much beyond the scope of this paper to model the equation of state or evolutionary development of the planetary core. Presumably it underwent considerable compression as the p-Sat envelope of mass $M_{\text {env }} \approx$ $85 M_{\oplus}$ gathered around it and underwent its own gravitational contraction to a progressively denser state. The total mass of the $\mathrm{p}$-Sat cloud for equatorial radius $R_{\mathrm{e}}$ is $M_{\mathrm{e}}=M_{\text {core }}+M_{\text {env }}$. The initial equatorial radius of the p-Sat cloud is taken to be $R_{\mathrm{e}, \mathrm{i}}=30 R_{\text {Sat }}$. Prentice (2005a) has proposed that the large water ice content of Tethys is due to the eviction of $\mathrm{H}_{2} \mathrm{O}$ from the planetary core once the cloud radius $R_{\mathrm{e}}$ has shrunk below $5 R_{\text {Sat }}$. In the case of a core of mass $10 M_{\oplus}$, it is necessary that $25 \%$ of the water content of the core be evicted in order to explain the mean density of Tethys.

Let $\rho_{\text {core }}=\rho_{\text {core }}\left(R_{\mathrm{e}}\right)$ denote the core density corresponding to cloud equatorial radius $R_{\mathrm{e}}$. A satisfactory model for the core-envelope system is achieved if it is assumed that the initial mass density of the core is $\rho_{\text {core }}\left(30 R_{\text {Sat }}\right)=$ $0.01 \mathrm{~g} \mathrm{~cm}^{-3} \cdot \rho_{\text {core }}$ then increases as a simple power of $R_{\mathrm{e}}$ to the value $1.0 \mathrm{~g} \mathrm{~cm}^{-3}$ at $R_{\mathrm{e}}=5 R_{\text {Sat }}$ and then on up to $3.25 \mathrm{~g} \mathrm{~cm}^{-3}$ at $R_{\mathrm{e}}=3 R_{\text {Sat }}$. It is assumed that the homologous contraction of the p-Sat cloud, dictated by the constancy of the parameters $\beta_{0}, \theta_{0}$, and $F_{\mathrm{s}}$ terminates at radius $R_{\mathrm{e}, \text { homog }}=$ $3.0 R_{\text {Sat }}$. Below that radius the surface temperature $T_{\mathrm{e}}$ of the cloud is assumed to pass smoothly to an end value of $325 \mathrm{~K}$ at radius $R_{\mathrm{e}, \text { end }}=1.5 R_{\mathrm{Sat}}$. The turbulence parameter $\beta_{0}$ and polytropic index $n_{\mathrm{t}}$ of the outer superadiabatic layer of the p-Sat cloud also decline in a controlled manner as the final cloud radius is approached. When $\beta \rightarrow 0, n_{\mathrm{t}}$ changes linearly with $\beta$ to a final value $n_{\text {ad }}$. This is the adiabatic value of the gas polytropic index. The decline in turbulent stress causes the geometric spacing between newly shed gas rings to steadily diminish. The Mimas gas ring is shed at $2.777 R_{\text {Sat }}$. At radius $R_{\mathrm{e}}=2.56 R_{\text {Sat }}$, all shedding of discrete gas rings ceases. Thereafter the cloud remains rotationally stable by shedding mass continuously, so forming an inner gas disc. Eventually, even disc shedding terminates when the moment-of-inertia factor of the $\mathrm{p}$-Sat cloud rises sufficiently for the rotational velocity at the equator to drop below the local Keplerian value, i.e. $v_{\mathrm{Kep}}=\left(G M_{\mathrm{e}} / R_{\mathrm{e}}\right)^{1 / 2}$.

\section{Predicted Bulk Chemical Composition of Rhea and a Model for its Thermal Evolution}

\subsection{Computed Bulk Chemical Compositions of the Satellites}

Table 3 gives the basic physical and chemical properties of the system of gas rings shed by the p-Sat cloud. As noted above, $R_{n, \mathrm{i}}$ denotes the initial mean orbital radii of the system of gas rings $(n=0,1,2,3 \ldots)$, measured in units of Saturn's present equatorial radius $R_{\mathrm{Sat}}$. This Table gives the temperature $T_{n}{ }^{*}$ of each gas ring at the time it is shed by the cloud, as well as the condensation temperatures $T_{i}$ of the principal ice species $\{i\}$. These are computed for the mean orbit gas pressures $p_{n}$. Figure 1 shows $T_{n}{ }^{*}$ and the quantities $T_{\mathrm{H}_{2} \mathrm{O}}, T_{\mathrm{NH}_{3}}$, etc., plotted as continuous functions of the present orbital distance $R_{n}$ of a locally detached gas ring. Allowance has been made in this plot for orbital expansion due to mass loss of the p-Sat cloud. $T_{\mathrm{NH}_{3}}$ is the condensation temperature of pure $\mathrm{NH}_{3}$ ice.

Table 4 gives the mass fractions $X_{i}$ of the principal chemical constituents for each gas ring. $X_{\mathrm{NH}_{3}}$ refers to the total of both pure $\mathrm{NH}_{3}$ ice and $\mathrm{NH}_{3} \cdot \mathrm{H}_{2} \mathrm{O}$. The latter substance is, however, either absent or totally negligible in all moons except Dione. $X_{\mathrm{CH}_{4}}$ is the mass of $\mathrm{CH}_{4}$ ice. This condenses initially as methane clathrate hydrate, $\mathrm{CH}_{4} \cdot 5.75 \mathrm{H}_{2} \mathrm{O}$. It is assumed now to exist as a free ice in the satellite interior. The condensation locus for argon clathrate hydrate is not included in Figure 1 since $T_{n}{ }^{*}$ everywhere exceeds $T_{\mathrm{Ar} \cdot 5.67 \mathrm{H}_{2} \mathrm{O}}$. At Hyperion's orbit, $T_{\mathrm{Ar} \cdot 5.67 \mathrm{H}_{2} \mathrm{O}}$ is $62 \mathrm{~K}$ and at $R_{n, \mathrm{i}}=17 R_{\mathrm{Sat}}$ it is $66 \mathrm{~K}$. The second- and third-last columns of Table 4 list the rock density $\rho_{\text {rock }}$ and the mean density of the condensate $\rho_{\text {cond }}$. These quantities are calculated at a pressure of $0.1 \mathrm{MPa}$ and temperature $T_{\text {Sat }}=76 \mathrm{~K}$ at Saturn's orbit. The last column of the Table gives the observed mean 
density of the satellite at distance $R_{n}$ (Jacobson 2004; Jacobson et al. 2005; Rappaport et al. 2005).

\subsection{Discussion of the Ammonia-Rich Bulk Chemical Composition of Rhea}

The remainder of this paper will be devoted to constructing a viable model for Rhea. The precise mass fractions of the bulk constituents of the Rhea condensate are hydrated rock (0.3853), water ice (0.3946), and pure $\mathrm{NH}_{3}$ ice $(0.2201)$. The most interesting feature of this mix is the high proportion of $\mathrm{NH}_{3}$ ice relative to $\mathrm{H}_{2} \mathrm{O}$ ice. If normal solar abundances were to have been assumed for the gas ring from which Rhea condensed, then Table 1 would yield an $\mathrm{NH}_{3}$ to $\mathrm{H}_{2} \mathrm{O}$ mass percent ratio of 15:85. Instead the ratio is 36:64. That is, $\mathrm{NH}_{3}$ makes up more than one-third of the ice mass. It cannot be ignored. The physical reason for the high proportion of $\mathrm{NH}_{3}$ resides in the proposed four-fold depletion of the abundance of rock and $\mathrm{H}_{2} \mathrm{O}$ ice relative to solar abundances within the residual gas of the proto-Solar gas ring at Saturn's orbit. This process was discussed in Section 2. The depletion comes about through the condensation of these species and their subsequent isolation from the gas in forming Saturn's core. The principal chemical constituents of the rock are $\mathrm{SiO}_{2}$ (mass fraction 0.2795), $\mathrm{Mg}(\mathrm{OH})_{2}(0.2760)$, FeS/NiS (0.1943), $\mathrm{Fe}_{3} \mathrm{O}_{4}(0.1010), \mathrm{Fe} / \mathrm{Ni}(0.0507), \mathrm{Ca}_{2} \mathrm{MgSi}_{2} \mathrm{O}_{7}$ (0.0391), $\mathrm{MgAl}_{2} \mathrm{O}_{4}$ (0.0298), and $\mathrm{NaOH} / \mathrm{KOH}$ (0.0114). The remaining constituents have mass fractions each less than 0.01 . The rock is strongly hydrated and has mean density at $298.15 \mathrm{~K}$ and $0.1 \mathrm{MPa}$ pressure of $3.1253 \mathrm{~g} \mathrm{~cm}^{-3}$. I note here that the majority of the numerical results in this paper are reported to four or more significant digit accuracy. This is to enable a useful comparison with the results of future computations where different compositional mixes of rock and ices may apply. The greatest uncertainty in the input data lies in the estimates of the solar elemental abundances, especially those of oxygen and nitrogen. Oxygen has an abundance uncertainty of $12 \%$ and nitrogen one of 29\% (Lodders 2003).

\subsection{Computation of the Thermal State of Rhea}

A computational code that was designed to model the thermal evolution of the Galilean moons of Jupiter has been applied to Rhea (Prentice 2001). It is assumed that the only heat source within the satellite is that generated by the radioactive decay of ${ }^{40} \mathrm{~K},{ }^{232} \mathrm{Th},{ }^{235} \mathrm{U}$, and ${ }^{238} \mathrm{U}$ in the rock. Tidal heating is ignored at this stage even although it is possible that the dynamical capture of Titan from a solar orbit (Prentice 1984, 2004a, 2004b) may have greatly stirred the orbital motion of Rhea in the beginning (R. A. Mardling, private communication). The present eccentricity of Rhea is so small that tidal dissipation is practically zero (cf. Peale 1999).

Figure 2 shows the temperature distribution within Rhea as a function of fractional satellite radius $r / R_{\mathrm{Rhea}}$ at a set of key times during the course of the thermal evolution to solar age (4600 Myr). At time $t=0$, the satellite is assumed to be chemically uniform throughout its interior and have uniform temperature equal to the present day mean surface value, namely $T_{\text {surf }}=T_{\text {Sat }}=$ $76 \mathrm{~K}$. The profiles in Figure 2 are the solution of the heat diffusion equation assuming that all heat transfer occurs only by means of conduction. The thermal conductivity of $\mathrm{NH}_{3}$ ice is modelled empirically from the data of Krupskii, Manzhely, and Koloskova (1968). It is a temperaturedependent quantity, as is the case for the conductivities of the other solid constituents of the Rhea compositional mix. Provided no melting takes place during the evolution, we see that the present day satellite is very cold throughout its interior. The warmest point is at the centre, where $T_{\mathrm{c}}(4600$ Myr) $=112 \mathrm{~K}$.

\subsection{Examining the Possibility of Melting of Ice}

Consider now the important question of whether any melting of ice takes place at some point during the thermal evolution to present age. The melting temperatures $T_{\mathrm{m}, i}$ of $\mathrm{H}_{2} \mathrm{O}$ ice $(i=1)$ and $\mathrm{NH}_{3} \cdot 2 \mathrm{H}_{2} \mathrm{O}$ (ammonia dihydrate $(\mathrm{ADH}), i=2$ ) ice are shown in Figure 2. Both of these quantities are functions of pressure $p$ and hence of the fractional radius $r / R_{\text {Rhea }}$. For ammonia dihydrate, Hogenboom et al. (1997) find

$T_{\mathrm{m}, 2}(p)[\mathrm{K}]=176.29+2.43 \times 10^{-2} p-7.7583 \times 10^{-3} p^{2}$

where the unit of $p$ is MPa. At the centre of the satellite, the thermal code gives $p_{\mathrm{c}}=123.4 \mathrm{MPa}$ and $T_{\mathrm{m}, 2}=$ $178 \mathrm{~K}$. Not shown in Figure 2 is $T_{\mathrm{m}, 3}$ for pure $\mathrm{NH}_{3}$ ice. This is everywhere about $20 \mathrm{~K}$ above that of the dihydrate ice. However, when $\mathrm{H}_{2} \mathrm{O}$ and $\mathrm{NH}_{3}$ coexist, melting first occurs at the eutectic point for the mixture of these ices. This is the temperature $T_{\mathrm{m}, 2}$.

It is clear from Figure 2 that if the possibility of convective heat transfer through solid-state creep of ice is ignored, melting will take place for fractional radii $r / R_{\mathrm{Rhea}} \leq 0.65$. The maximum melting radius is attained after about 500 Myr. Ellsworth \& Schubert (1983) were the first to explicitly model convective heat transfer in icy satellites. According to these authors, the Rayleigh number quickly rises above the critical value of approximately 1100 required for the onset of convection in a Rhea-sized radiogenically heated icy satellite. Even so, convection cannot start until the temperature exceeds a fixed fraction $f_{\text {creep }}$ of the local melting temperature $T_{\mathrm{m}}$. The curve marked $T_{\text {creep }}$ in Figure 2 is the solid-state creeping temperature of $\mathrm{H}_{2} \mathrm{O}$ ice for the case $f_{\text {creep }}=0.7$. Ellsworth \& Schubert (1983) adopted $f_{\text {creep }}=0.6$. Prentice \& Freeman (1999) found that a higher value is needed if we are to account for the existence of a sub-surface layer of liquid $\mathrm{ADH}$ in Callisto.

In the case of Callisto, $\mathrm{NH}_{3}$ ice makes up only about $9 \%$ of the ice mass, so the rheology is controlled by the properties of $\mathrm{H}_{2} \mathrm{O}$ ice. For the model of Rhea proposed here, however, $\mathrm{NH}_{3}$ comprises approximately $36 \%$ of 


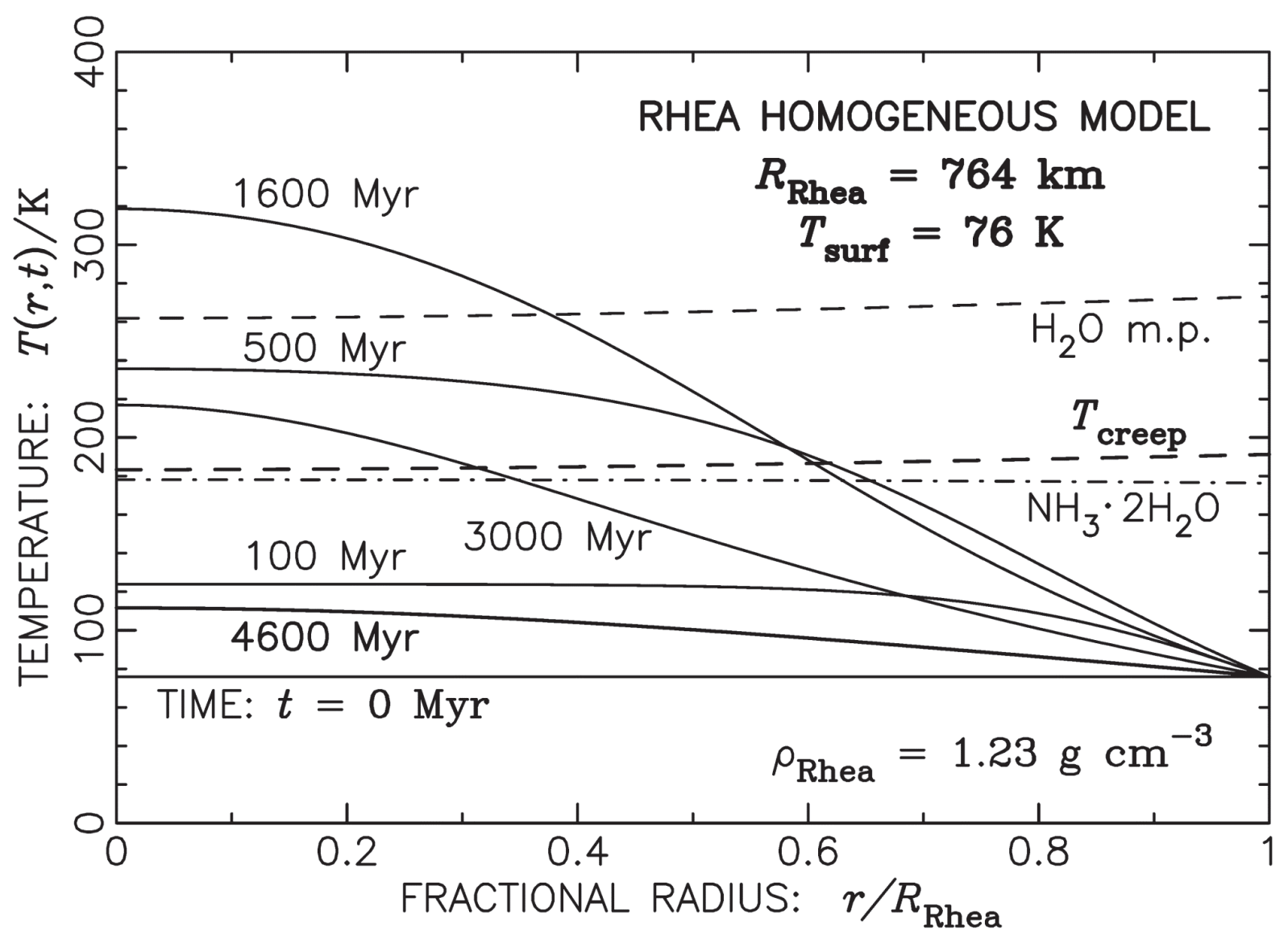

Figure 2 Internal temperature profiles of a homogeneous Rhea-sized satellite comprised of rock, $\mathrm{H}_{2} \mathrm{O}$ ice, and $\mathrm{NH}_{3}$ ice in the fractional mass proportions $0.3853,0.3946$, and 0.2201 . The profiles of temperature versus fractional radius $r / R_{\text {Rhea }}$ are shown at various times $t$ during the course of the satellite's thermal evolution to present age (4600 Myr). All heat is derived from the decay of radioactive nuclides. The melting temperature of $\mathrm{H}_{2} \mathrm{O}$ ice and that of ADH are shown by the broken lines. They depend on pressure and hence on $r / R_{\text {Rhea }}$. The satellite becomes locally unstable towards solid-state convection when the temperature $T(r, t)$ rises through the creep value $T_{\text {creep }}=$ $0.7 T_{\mathrm{m}, \mathrm{H}_{2} \mathrm{O}}$. The contribution of solid-state convection to the heat transfer rate has not been included here.

the ice mass. This closely matches the dihydrate proportion $(32.1 \%)$. This means that the solid-state convection is now controlled by the rheology of the dihydrate ice. The true creep temperature will thus be closer to $140 \mathrm{~K}$. We can be fairly certain, therefore, that none of the interior of Rhea will undergo melting at any stage during its thermal evolution to solar age if all heating is derived solely from radiogenic decay. The present temperature distribution within Rhea may thus be reasonably assumed to be very similar to the $t=$ 4600 Myr profile shown in Figure 2.

\section{Structural Models of Rhea}

\subsection{The Controlling Equations and the Equations of State}

It remains to construct a self-consistent model for Rhea, based on the computed temperature profile found in Section 4. This task is performed using a computational code that was also developed to numerically model the interior structures of the Galilean satellites (Prentice 1996a, 1996b, 2001).

The distribution of pressure $p=p(r)$ with radius $r$ in a spherically symmetric satellite of surface radius $R_{\mathrm{S}}$ is controlled by the hydrostatic support equation

$$
\frac{\mathrm{d} p}{\mathrm{~d} r}=-\frac{\rho G M(r)}{r^{2}}
$$

Here $\rho=\rho(r)$ is the local mean density and $M(r)$ is the mass interior to radius $r$. For a solid composed of $N$ chemical species each of mass fraction $X_{j}$ and density $\rho_{j}$, the mean density is given by

$$
\frac{1}{\rho}=\sum_{j=1}^{N} \frac{X_{j}}{\rho_{j}}
$$

Each of the quantities $\rho_{j}$ depends on both $p$ and the local temperature $T$. That is, it is necessary to know the equation of state (EOS) of each of the chemical constituents of the solid. In the case of most rocky minerals, an excellent 
representation of the thermal and compressional behaviour is achieved by fitting a Murnaghan EOS. We have

$$
\rho_{j}(p, T)=\rho_{j, 0.1}(T)\left[1+\frac{K_{j, 0.1}^{\prime}}{K_{j, 0.1}}(p-0.1)\right]^{\alpha} \quad \alpha=\frac{1}{K_{j, 0.1}^{\prime}}(6)
$$

Here $K_{j, 0.1}$ and $K_{j, 0.1}^{\prime}$ denote the bulk modulus and its first pressure derivative at $0.1 \mathrm{MPa}$ pressure and $\rho_{j, 0.1}(T)$ is the mean density, also at $0.1 \mathrm{MPa}$. The main advantage of this separable representation for the density is that one can utilize the great body of experimental data on the thermal expansion of solids that exists for most minerals at $0.1 \mathrm{MPa}$ pressure.

The EOS for water ice is extremely complex owing to the existence of the many high-pressure phases of this ice. Empirical representations for the densities $\rho_{\mathrm{H}_{2} \mathrm{O}, j}(p, T)$ of each phase $j$ are assembled in Prentice (2001). For $\mathrm{NH}_{3}$ ice the following Murnaghan-style EOS has been constructed by combining the thermal expansion data of Croft, Lunine, \& Kargel (1988) and the compressional data of Stewart (1960):

$$
\begin{aligned}
\rho_{\mathrm{NH}_{3}, 0.1}(T)\left[\mathrm{g} \mathrm{cm}^{-3}\right] & =0.8659 \exp \left(-4.198 \times 10^{-7} T^{2.2207}\right) \\
A_{1}\left[\mathrm{MPa}^{-1}\right] & =(1.495+0.001 T) \times 10^{-4} \\
B_{1}\left[\mathrm{MPa}^{-2}\right] & =\left(4.5066-7.778 \times 10^{-3} T\right) \times 10^{-8} \\
K_{\mathrm{NH}_{3}, 0.1}^{\prime} & =\left(2 B_{1} / A_{1}^{2}\right)-1 \\
K_{\mathrm{NH}_{3}, 0.1}[\mathrm{MPa}] & =1 / A_{1}
\end{aligned}
$$

The EOS of ADH ice has been investigated by Croft et al. (1988), Hogenboom et al. (1997), and Fortes et al. (2003). There are two distinct pressure phases of this ice. The transition from the low-pressure phase ADH I to the high-pressure phase $\mathrm{ADH}$ II depends on temperature but always exceeds about $320 \mathrm{MPa}$. Since the ice pressure in Rhea never exceeds $150 \mathrm{MPa}$, all ADH ice exists as phase I. The computations of Fortes et al. (2003) give:

$$
\begin{aligned}
\rho_{\mathrm{ADHI}, 0.1}(T)\left[\mathrm{g} \mathrm{cm}^{-3}\right] & =0.9917 \exp \left(-1.1757 \times 10^{-7} T^{2.39}\right) \\
K_{\mathrm{ADHI}, 0.1}^{\prime} & =5.38 \\
K_{\mathrm{ADH}, 0.1}[\mathrm{MPa}] & =10670
\end{aligned}
$$

Insertion of these quantities into Eqn (6) yields the EOS for ADH I.

\subsection{Results and Discussion}

Five distinct models for Rhea have been constructed according to the choice of the various controlling parameters, which are listed in Table 5. These parameters include the global mass fractions $X_{i}$ of rock, $\mathrm{H}_{2} \mathrm{O}$ ice, and $\mathrm{NH}_{3}$ ice, and a new quantity $\zeta_{\mathrm{NH}_{3}}$. This defines an enhancement of the mass of $\mathrm{NH}_{3}$ relative to the cosmogonically derived value of 0.2201 . The five models are labelled H1, D, H2, H3, and H4, respectively. Models H1, $\mathrm{H} 2, \mathrm{H} 3$, and $\mathrm{H} 4$ have chemically homogeneous interiors. Model D is a differentiated structure having a central rocky core and icy mantle. Each satellite model consists of two zones of different temperature $T_{1}$ and $T_{2}$. For the homogeneous models, the boundary between these zones is the mass mid-point. For model D, $T_{1}$ and $T_{2}$ are the core and mantle mean pressures. The last column gives the transition pressure $p_{\mathrm{I} / \mathrm{II}}$ that defines the boundary between phase I and II of water ice. All quantities have been computed for the thermal profile at $4600 \mathrm{Myr}$.

\subsubsection{A First Homogeneous Satellite Model: H1}

This model has a uniform mixture of rock and ices in the proportions established from solar abundances and the depletion factor $\zeta_{\text {dep }}=0.25$ that was introduced in Section 2. The principal characteristics of the model are listed in Table 6 . Within the inner $17 \%$ of the satellite mass, all $\mathrm{H}_{2} \mathrm{O}$ ice is in the dense phase II state. This phase is $27 \%$ denser than phase I ice at the same temperature and pressure. Overall, the model is too dense compared to the observed density of Rhea, namely, $\rho_{\text {Rhea }}=1.23 \pm$ $0.02 \mathrm{~g} \mathrm{~cm}^{-3}$, the error being $2.5 \sigma$.

\subsubsection{A Differentiated Satellite Model: D}

This model assumes that all rock has settled to the centre following an unanticipated meltdown of all the ice. If such a meltdown did take place, perhaps as a result of powerful tidal action due to Titan, the liquid mantle would refreeze as a eutectic mixture forming a deep mantle of ADH ice of mass fraction 0.5811. This $\mathrm{ADH}$ mantle is surmounted by a thin crust of pure $\mathrm{NH}_{3}$ ice of mass fraction 0.00336 and thickness $13.5 \mathrm{~km}$. Coincidentally, the mean density of the model matches the value $1.33 \pm 0.10 \mathrm{~g} \mathrm{~cm}^{-3}$ found by the Voyager 1 spacecraft (Tyler et al. 1981). The Cassini data suggests that this model is no longer viable.

\subsubsection{A Second Homogeneous Model: H2}

This model attempts to match the observed mean density of Rhea by assuming that the actual mass of $\mathrm{NH}_{3}$ ice relative to rock and $\mathrm{H}_{2} \mathrm{O}$ ice is a factor

Table 5. Controlling parameters for the family of Rhea structural models

\begin{tabular}{lccccrrr}
\hline & $X_{\text {rock }}$ & $X_{\mathrm{H}_{2} \mathrm{O}}$ & $X_{\mathrm{NH}_{3}}$ & $\zeta_{\mathrm{NH}_{3}}$ & $T_{1}[\mathrm{~K}]$ & $T_{2}[\mathrm{~K}]$ & $p_{\mathrm{I} / \mathrm{II}}[\mathrm{MPa}]$ \\
\hline $\mathrm{H} 1$ & 0.3853 & 0.3946 & 0.2201 & 1.00 & 96 & 81 & 96.0 \\
$\mathrm{D}$ & 0.3853 & 0.3946 & 0.2201 & 1.00 & 100 & 81 & 87.8 \\
$\mathrm{H} 2$ & 0.3577 & 0.3664 & 0.2759 & 1.35 & 97 & 81 & 97.6 \\
$\mathrm{H} 3$ & 0.3853 & 0.3946 & 0.2201 & 1.00 & 96 & 81 & - \\
$\mathrm{H} 4$ & 0.3690 & 0.3780 & 0.2530 & 1.20 & 97 & 81 & - \\
\hline
\end{tabular}


Table 6. Structural Properties of the Rhea Models

\begin{tabular}{|c|c|c|c|c|c|}
\hline \multirow[t]{2}{*}{ Property } & \multicolumn{5}{|c|}{ Model } \\
\hline & H1 & $\mathrm{D}$ & $\mathrm{H} 2$ & H3 & $\mathrm{H} 4$ \\
\hline Central pressure $[\mathrm{MPa}]$ & 143.5 & 347.5 & 130.4 & 128.9 & 124.1 \\
\hline Rock core radius $[\mathrm{km}]$ & - & 417 & - & - & - \\
\hline Radius of dihydrate boundary $[\mathrm{km}]$ & - & 750 & - & - & - \\
\hline Ice I / Ice II boundary [km] & 410 & - & 356 & - & - \\
\hline Ice II / total $\mathrm{H}_{2} \mathrm{O}$ ice & 0.171 & - & 0.112 & - & - \\
\hline Central density $\left[\mathrm{g} \mathrm{cm}^{-3}\right]$ & 1.425 & 3.172 & 1.362 & 1.264 & 1.240 \\
\hline Surface density $\left[\mathrm{g} \mathrm{cm}^{-3}\right]$ & 1.248 & 0.860 & 1.209 & 1.249 & 1.225 \\
\hline Mean density $\left[\mathrm{g} \mathrm{cm}^{-3}\right]$ & 1.279 & 1.339 & 1.229 & 1.254 & 1.231 \\
\hline$C / M R^{2}$ & 0.394 & 0.324 & 0.396 & 0.3995 & 0.3995 \\
\hline
\end{tabular}

$\zeta_{\mathrm{NH}_{3}}=1.35$ times larger than the value suggested earlier. Now as mentioned earlier, the solar abundance of nitrogen has an uncertainty of $29 \%$. The $\zeta_{\mathrm{NH}_{3}}$ value for model $\mathrm{H} 2$ thus lies just outside the error range, but not significantly so. We need to bear in mind that the value of the depletion factor $\zeta_{\text {dep }}$ relating to the exhaustion of solids relative to gas in the proto-Solar gas ring is also uncertain, probably by about $20 \%$. That is, $\zeta_{\text {dep }}=0.25 \pm 0.05$. The $\mathrm{H} 2$ model thus cannot be ruled out on abundance grounds.

\subsubsection{Homogeneous Models without Water Ice Phase II: Models $\mathrm{H} 3$ and H4}

The only objection to model H1 was that it is too dense. Now this satellite model contains a large quantity of the dense phase II of $\mathrm{H}_{2} \mathrm{O}$ at its centre. As Ellsworth \& Schubert (1983) have noted, the formation of this material in the core of the cooling satellite should have caused the mean radius to slump by about $15 \mathrm{~km}$. In our model the estimated slump is around $5 \mathrm{~km}$. Even so, such shrinkage should have left prominent compressional features on the surface, such as networks of parallel ridges and troughs. Such features have so far not been observed, suggesting that the formation of phase II of water ice was somehow suppressed.

The crucial step now is the realization that because the ice in the present study consists of an intimate mixture of $\mathrm{H}_{2} \mathrm{O}$ and $\mathrm{NH}_{3}$ of comparable proportions, the formation of phase II of $\mathrm{H}_{2} \mathrm{O}$ ice may never take place. That is, the substantial and intimate intrusion of $\mathrm{NH}_{3}$ in the $\mathrm{H}_{2} \mathrm{O}$ ice may prevent the crystallization of the dense phases of water ice. This idea was first mooted by D. J. Stevenson, as a private communication in Consolmagno (1985). Further support that ammonia ice suppresses the formation of phase II of water ice is found in the data of Hogenboom et al (1997). In figure 3a of that paper there is no evidence for a discontinuity in the phase diagram of ADH ice near the pressure of $100 \mathrm{MPa}$, where pure water ice changes from phase I to II. The phase boundary separating liquid and solid is well behaved for pressures all the way from 0 to $320 \mathrm{MPa}$. A discontinuity in the slope of the phase boundary does occur at a triple point at $320 \mathrm{MPa}$. Here phase I of ADH ice transforms to the highpressure phase II, and water ice II first appears in coexistence with liquid. The pressure at the rock/ice boundary of model D is $103.5 \mathrm{MPa}$.

In addition, a recent paper by Fortes et al. (2003) also finds no evidence for a discontinuity in the equation of state of ADH in the pressure range 0-320 MPa. These authors suggest that there was a possible application of their work to Rhea and stated explicitly that only the low-pressure phase of ADH would be present. They did not present any model for Rhea. To date, the experimental evidence of two groups supports the contention that ammonia ice suppresses the formation of phase II of water ice.

The remaining structural models of Rhea do not possess any phase $\mathrm{II} \mathrm{H}_{2} \mathrm{O}$ ice, despite the fact that the pressure exceeds the $p_{\mathrm{I} / \mathrm{II}}$ transition pressure throughout much of the central region of the satellite. Model H3 assumes the cosmogonic bulk composition of rock, $\mathrm{H}_{2} \mathrm{O}$ ice, and $\mathrm{NH}_{3}$ ice, corresponding to the choice $\zeta_{\mathrm{NH}_{3}}=$ 1.0. For model $\mathrm{H} 4, \zeta_{\mathrm{NH}_{3}}=1.2$. The mean density of the $\mathrm{H} 3$ model is much closer to the observed value of Rhea. In fact it lies only just over $1 \sigma$ away. The best fit of all the Rhea models is $\mathrm{H} 4$. Here $\zeta_{\mathrm{NH}_{3}}$ is chosen so that the model mean density matches $\rho_{\text {Rhea }}$. The required increase in $\zeta_{\mathrm{NH}_{3}}$ from 1.0 to 1.2 is well inside the observational uncertainty of the solar nitrogen abundance. It is also compatible with the permitted range of the depletion factor, namely $\zeta_{\text {dep }}=0.25 \pm 0.05$.

Finally, since the compression of rock and ice is everywhere so modest, there is very little contrast between the central and surface densities of the satellite model. The temperature contrast between these regions is also small. It is predicted that the Cassini measurements taken during the Rhea- 1 close flypast of 2005 November 26 will find Rhea to be a cold and essentially chemically homogeneous structure. The predicted axial moment-of-inertia coefficient is

$$
\left[C / M R^{2}\right]_{\text {Rhea }}=0.399 \pm 0.004
$$




\section{Conclusions}

Theoretical and numerical models for the origin, bulk chemical composition and internal structure of Rhea have been put forward at the time of the Cassini spacecraft first close flyby. It is proposed that as a result of condensation and depletion of solids within the proto-Solar gas ring from which the $\mathrm{p}$-Sat gas cloud was derived, that this cloud was deficient by a factor $\zeta_{\text {dep }}=0.25$ in rock and $\mathrm{H}_{2} \mathrm{O}$ ice relative to solar abundance of these materials. Nitrogen, however, is present in nearly solar abundance proportions and, as a result, the mass percent ratio of $\mathrm{NH}_{3}$ to $\mathrm{H}_{2} \mathrm{O}$ in the p-Sat cloud is 36:64.

It is proposed that Saturn's mid-sized moons condensed from a concentric family of gas rings that were cast off at the equator of the contracting p-Sat cloud. A process of supersonic turbulent convection that has previously been applied successfully to calculate the properties of the system of gas rings shed by the proto-Solar and proto-Jovian clouds is used to determine the properties of the p-Sat gas rings. The temperatures $T_{n}$ of these rings vary closely with mean orbital distance $R_{n}(n=0,1,2,3 \ldots)$ according to $T_{n}=A / R_{n}$. If the constant of proportionality is chosen to account for the observed mean density of Enceladus, then Rhea condenses well inside the stability field of $\mathrm{NH}_{3}$ ice. The predicted bulk chemical composition by mass fraction is rock (0.3853), $\mathrm{H}_{2} \mathrm{O}$ ice $(0.3946)$, and $\mathrm{NH}_{3}$ ice $(0.2201)$. We refer to this as the 'cosmogonic' chemical mixture.

A family of numerical models for the thermal evolution and present-day interior structure of Rhea have been constructed. It is not possible to construct a chemically differentiated satellite model whose mean density matches that of Rhea. Homogeneous models can, however, be constrained to match the observed mean density provided that it is assumed that the mass fraction of $\mathrm{NH}_{3}$ ice exceeds the cosmogonic value by a factor $\zeta_{\mathrm{NH}_{3}}=1.20-1.35$. Next it is proposed that the large quantity of $\mathrm{NH}_{3}$ ice, relative to solar abundance expectation, may have prevented the formation of the dense phase $\mathrm{II}$ of $\mathrm{H}_{2} \mathrm{O}$ ice near the satellite centre. This may explain the observed dearth of compressional features on the surface. Such features would have otherwise formed as the central regions of the moon cooled following the subsidence of radiogenic heating.

The favoured model of Rhea has a chemically uniform interior and is very cold. The central temperature exceeds the surface value by barely $40 \mathrm{~K}$. The satellite is nearly isodense and the predicted value of the axial moment-of-inertia factor is $\left[C / M R^{2}\right]_{\text {Rhea }}=0.399 \pm 0.004 . \mathrm{NH}_{3}$ ice is unstable at Saturn's distance from the Sun, except near the polar regions of a satellite. Perhaps the Cassini Orbiter will discover indirect evidence for $\mathrm{NH}_{3}$ through the sublimative escape of this ice from the outer layers, especially near the equatorial zones. Wasting of $\mathrm{NH}_{3}$ would weaken the residual soil, so making the edges of craters soft and prone to landslides. It will be exciting to learn what Cassini discovers.

\section{Acknowledgements}

The author thanks P. D. Godfrey, R. A. Mardling, and N. J. Rappaport for helpful discussions. G. W. and C. Null,
C. and M. Savalla, and E. M. Standish Jr offered generous hospitality in Pasadena. S. Campbell, L. Mayer, C. Morgan, S. Morton, A. Thorne, and C. Wilson provided valuable technical support. Lastly, I thank G. Consolmagno for a constructive review of the manuscript.

\section{References}

Anderson, J. D., Colombo, G., Esposito, P. B., Lau, E. L., \& Trager, G. B. 1987, Icar, 71, 337

Asplund, M., Grevesse, N., \& Sauval, A. J. 2005, in ASP Conf. Ser. 336: Cosmic Abundances as Records of Stellar Evolution and Nucleosynthesis (Eds Bash, F. N., \& Barnes, T. G.), 25 (San Francisco: ASP)

Bahcall, J. N., Pinsonneault, M. H., \& Basu, S. 2001, ApJ, 555,990

Castillo, J. C. 2005, LPI, 36, 2243

Clark, R. N., Brown, R. H., Owensby, P. D., \& Steele, A. 1984, Icar, 58, 265

Consolmagno, G. J. 1985, Icar, 64, 401

Consolmagno, G. J., \& Lewis, J. S. 1978, Icar, 34, 280

Croft, S. K., Lunine, J. I., \& Kargel, J. 1988, Icar, 73, 279

Ellsworth, K., \& Schubert, G. 1983, Icar, 54, 490

Fortes, A. D., Wood, I. G., Brodholt, J. P., \& Vocadlo, L. 2003, Icar, 162,59

Hogenboom, D. L., Kargel, J. S., Consolmagno, G. J., Holden, T. C., Lee, L., \& Buyyounouski, M. 1997, Icar, 128, 171

Jacobson, R. A. 2004, AJ, 128, 492

Jacobson, R. A., Antreasian, P. G., Bordi, J. J., Criddle, K. E., Ionasescu, R., Jones, J. B., Mackenzie, R. A., Meek, M. C., Pelletier, F. J., Roth, D. C., Roundhill, I. M., \& Stauch, J. R. 2005, BAAS, 36, 524

Krupskii, I. N., Manzhely, V. G., \& Koloskova, L. A., 1968, PSS, 27, 263

Lebofsky, L. A. 1975, Icar, 25, 205

Lewis, J. S. 1972, Icar, 16, 241

Lodders, K. 2003, ApJ, 591, 1220

Lupo, M. J., \& Lewis, J. S. 1979, Icar, 40, 157

Peale, S. J. 1999, ARA\&A, 37, 533

Prentice, A. J. 1973, A\&A, 27, 237

Prentice, A. J. R. 1978a, in The Origin of the Solar System (Ed. Dermott, S. F.), p. 111 (New York: Wiley)

Prentice, A. J. R. 1978b, M\&P, 19, 341

Prentice, A. J. R. 1984, EM\&P, 30, 209

Prentice, A. J. R. 1996a, EM\&P, 73, 237

Prentice, A. J. R. 1996b, PhyLA, 213, 253

Prentice, A. J. R. 2001, EM\&P, 87, 11

Prentice, A. J. R. 2004a, BAAS, 36, 780

Prentice, A. J. R. 2004b, BAAS, 36, 1116

Prentice, A. J. R. 2005a, LPI, 36, 2378

Prentice, A. J. R. 2005b, BAAS, 37, 729

Prentice, A. J., \& Freeman, J. C. 1999, TAGU, 80, F607

Prentice, A. J. R., \& Dyt, C. P. 2003, MNRAS, 341, 644

Prinn, R. G., \& Fegley, B. 1981, ApJ, 249, 308

Rappaport, N. J., Iess, L., Tortora, P., Asmar, S. W., Somenzi, L., Anabtawi, A., Barbinis, E., Fleischman, D. U., \& Goltz, G. L. 2005, BAAS, 37, 704

Ross, J. E., \& Aller, L. H. 1976, Sci, 191, 1223

Smith, B. A., Soderblom, L., Beebe, R., Boyce, J., Briggs, G., Bunker, A., Collins, S. A., Hansen, C. J., Johnson, T. V., Mitchell, J. L., Terrile, R. J., Carr, M., Cook, A. F., Cuzzi, J., Pollack, J. B., Danielson, G. E., Ingersoll, A., Davies, M. E., Hunt, G. E., Masursky, H., Shoemaker, E., Morrison, D., Owen, T., Sagan, C., Veverka, J., Strom, R., \& Suomi, V. 1981, Sci, 212, 163

Stewart, J. W. 1960, JChPh, 33, 128

Tyler, G. L., Eshleman, V. R., Anderson, J. D., Levy, G. S., Lindal, G. F., Wood, G. E., \& Croft, T. A. 1981, Sci, 212, 201 